\title{
Polio Endgame: How India Beat the Monster*
}

Anuja Madhekar

India has had an incredible journey of becoming polio-free in a span of two decades. The conventional vaccination strategies and innovative approaches to ensure that every child in the remotest of the villages of India gets immunized have been an achievement in itself. The year $\mathbf{2 0 2 0}$ marks six years of living a dream that a sprawling country like India had harboured for long, and the efforts to keep the country polio-free need to continue until this dreadful disease gets eradicated globally.

"Hope lies in dreams, in the imagination and in the courage of those who dare to make dreams into reality", said Dr Jonas Salk, the man who successfully developed the first polio vaccine and who rightly deserves the credit for beating the once dreadful disease called poliomyelitis that has left millions of children paralysed all over the world. India too harboured the dream of beating poliomyelitis. This disease had created havoc in the early 20th century, paralysing thousands of children globally. But with the advent of scientific research, the introduction of an effective polio vaccine brought this disease under control in the developed countries. However, the developing countries world over still failed to see this disease as a threat to their future generations.

\section{About Poliomyelitis}

Poliomyelitis is a highly communicable viral disease generally known to be associated with poor sanitary conditions and lack of clean drinking water which are the common problems of communities below the poverty line. The causative agent of this disease is an RNA virus belonging to the group of enteroviruses. There are three known types of the wild poliovirus, namely Type

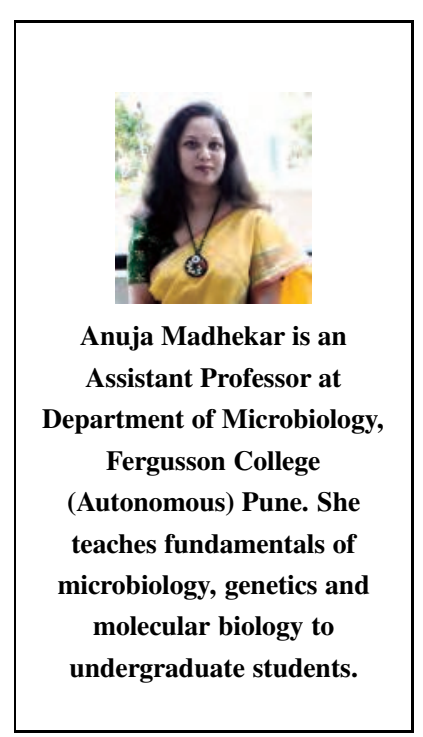

Keywords

Poliomyelitis, WHO, GPEI, VAPP, endemic, vaccination, surveillance, Covid 19.

${ }^{*}$ Vol.25, No.12, DOI: https://doi.org/10.1007/s12045-020-1095-7 
Poliovirus is known to spread predominantly through the faecal-oral route of transmission.

However, there is epidemiological evidence of respiratory transmission as well. On entering the human body, it multiplies at the site of implantation in the pharynx or intestine and soon invades the central nervous system causing permanent paralysis.
I, Type II and Type III. This virus is known to spread predominantly through the faecal-oral route of transmission [1]. However, there is epidemiological evidence of respiratory transmission as well [2]. On entering the human body, it multiplies at the site of implantation in the pharynx or intestine and soon invades the central nervous system causing permanent paralysis. While there is no cure for this disease, immunization can certainly help in preventing it.

\section{Global Polio Eradication Initiative}

In 1988, national governments and four organizations - the World Health Organization (WHO), Rotary International, Centre for Disease Control, and UNICEF came together to formulate the 'Global Polio Eradication Initiative' (GPEI) which started working with a single goal to eradicate polio. Later, the Bill \& Melinda Gates Foundation and Gavi, the Vaccine Alliance joined in [9]. GPEI since then has been supporting nations to execute various strategies for eradicating polio. These strategies include organizing mass immunization campaigns and strengthening global surveillance to prevent re-infection in the polio-free regions.

\section{History of Polio in India}

In the same year as that of the birth of GPEI, WHO signed a treaty with many countries asking them to participate in the global fight to eradicate polio. India was one of the signatories. The target year set by the World Health Assembly (WHA) to eradicate polio in India was 2000; however, India could not succeed in doing so. There were many reasons for this failure. One of the many reasons was the ignorance of Indian policymakers because of which nothing much happened on the ground post this treaty signing [3]. Earlier, in 1994, the then local government of New Delhi decided to take up the challenge and conducted a pilot polio immunization activity in Delhi targeting one million children up to the age of three. This idea slowly started to accelerate and boosted the confidence of our health ministries that polio could be 


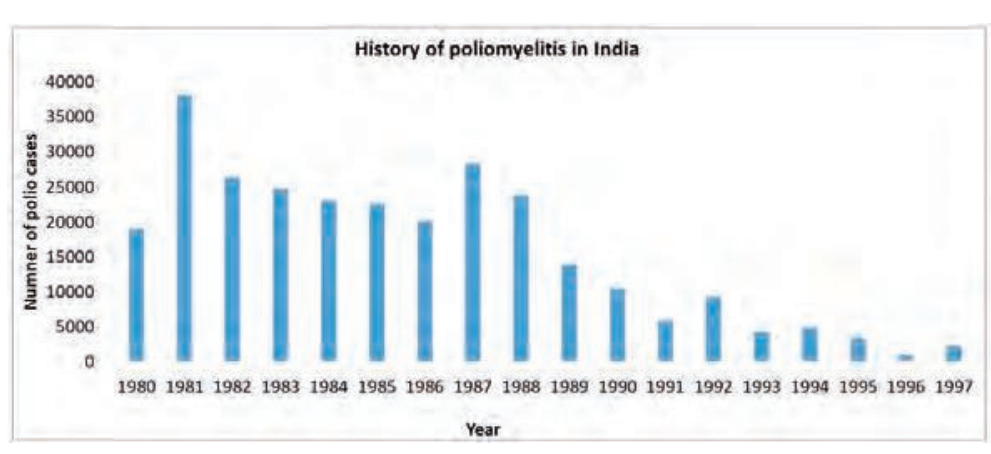

eradicated if we proceed in the right direction. A year later, India launched its first nationwide polio immunization campaign in 1995 (Figure 1). The tradition of 'National Immunization Day', to immunize all children up to the age of three years was started. Despite the tangible success that followed the mass immunization campaigns, the national government could not turn a blind eye towards the failure to vaccinate every child owing to the complexities of our country in terms of topography, huge and diverse population, poorly managed healthcare system, the existence of mobile families, etc. This failure was the second reason for India not being able to achieve the eradication target. Another major lacuna in the whole eradication strategy was a severe lack of surveillance which should have gone hand in hand with the immunization programs. This further reduced our chances to become polio-free by 2000. This pushed our government to further collaborate with WHO, and India's 'National Polio Surveillance Project' (NPSP) was initiated in 1997. This helped India's polio campaign to gain momentum.

\section{Vaccination Strategies of India}

The polio vaccine is available in two forms-inactivated polio vaccine (IPV) which consists of the killed form of the virus, whereas the oral polio vaccine (OPV) consists of a live virus whose ability to cause disease and establish infection is lost. Both types of these vaccine preparations when introduced into the body can prep our immune system to fight the virus in case of an infection.
Figure 1. History of polio in India [12].

India launched its first nationwide polio immunization campaign in 1995. The tradition of 'National Immunization Day', to immunize all children up to the age of three years was started.

The polio vaccine is available in two forms-inactivated polio vaccine (IPV) which consists of the killed form of the virus, whereas the oral polio vaccine (OPV) consists of a live virus whose ability to cause disease and establish infection is lost. Both types of these vaccine preparations when introduced into the body can prep our immune system to fight the virus in case of an infection. 
IPV has to be administered through injection by a trained health worker, while OPV can be administered orally by anybody.
Another major difference between OPV and IPV is that IPV has to be administered through injection by a trained health worker, while OPV can be administered orally by anybody. A trained health worker is not required for its administration. Now, considering the complexities of our country as mentioned earlier, OPV was concluded to be a more feasible option. However, this proved to be the biggest reason for the delay in achieving the eradication target of the year 2000 set by WHA as it led to growing cases of vaccine-associated paralytic poliomyelitis (VAPP), the details of which are elaborated in the next section at length. India had already started conducting National Immunization Day (NID) twice a year as per WHO recommendation. Simultaneously, many innovative approaches were also adopted to deal with polio such as conducting the 'Sub National Immunization Day' (SNID) annually in high-risk states like Uttar Pradesh, West Bengal, and Bihar. In almost all the states, polio campaigns were launched with setting up of vaccination booths in every neighbourhood of the city, village, and town. In Uttar Pradesh which was a polio-endemic state, an unconventional idea was implemented where a children's brigade called the 'Bulawa Toli' (or 'Calling Troupe') was asked to parade through the neighbourhood waving flags, blowing whistles, and calling out polio vaccination slogans, inviting younger children to the booth to receive OPV [8]. This implementation of pulse polio initiative at the designated booths on NIDs and SNIDs continued till 1999, but there was still a major chunk of the rural population that could not make it to these booths due to various reasons. This became another contributing factor for missing the eradication target of the year 2000. Hence a 'House to House' strategy was implemented, wherein, the front-line health workers would travel and walk miles together into the remotest regions to administer OPV to the children in their homes. Another innovative strategy adopted by the front-line workers to target the mobile families in our country was to immunize them at bus stations, train stations, and construction sites as well. These strategies were necessary because children all over the country needed to be vaccinated at the same time to prevent transmission of the virus, which is 
passed downstream by a vaccinated child as it happens routinely with OPV. This would help develop herd immunity in the population, which was very much desirable to eradicate polio.

\section{Vaccine Associated Paralytic Poliomyelitis (VAPP): A Grow- ing Concern}

Amidst these small victories, many Indian researchers [4] pointed out the danger of vaccine-associated paralytic poliomyelitis (VAPP) lurking around. VAPP is the result of the live attenuated virus from OPV preparation undergoing a back mutation causing neurotoxicity and development of paralytic polio. NPSP data had confirmed such cases based on acute flaccid paralysis (AFP) occurrence within 4 to 40 days of receiving OPV and detection of vaccine-derived poliovirus (VDPV) which is a result of a mutation in the vaccine virus [5], along with its detection in the stool samples of these patients. Reports state that the majority of the VAPP cases were a result of OPV administration since 1999. Although the Government of India (GOI) does not consider VAPP cases in the polio count of surveillance data justifying it to be a sporadic event, still many experts in the field suggested few measures to be adopted to speed up the end game strategy. Nonetheless, GOI took the first step towards addressing this issue of VAPP by deciding to switch over from the usage of trivalent OPV (tOPV) containing Type 1, 2 and 3 strains to bivalent OPV (bOPV) containing Type 1 and 3. This major decision of switching over was based on the fact that wild poliovirus Type 2 was eliminated back in 1999, and the continued usage of tOPV was responsible for about $40 \%$ of the VAPP cases. This was evident from the circulating vaccine-derived poliovirus (cVDPV), a subtype of VDPV isolated from the stool samples of VAPP patients. A meeting was held by the Ministry of Health \& Family Welfare in December 2015 in accordance with the National Switch Plan to execute this switch on 25th April 2016. Post this, Type 2 virus was completely excluded from the OPV preparation, and the remaining stocks of tOPV were disposed of except in some facilities like NIV, Pune, where the stock was to be retained for re-
Vaccine-associated paralytic poliomyelitis (VAPP) is the result of the live attenuated virus from OPV preparation undergoing a back mutation causing neurotoxicity and development of paralytic polio. 
Figure 2. Current scenario of polio in India [12].

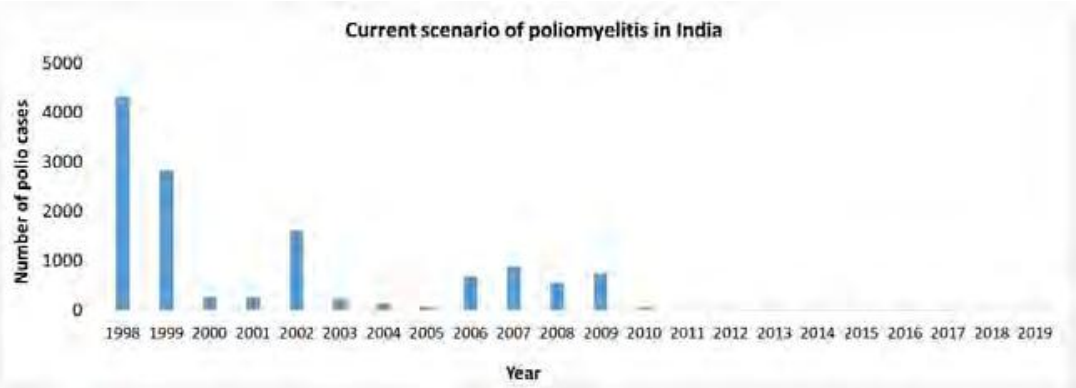

search purposes. [6]. Another measure suggested by the Strategic Advisory Group of Experts (SAGE) and the Indian Academy of Pediatrics (IAP) to deal with VAPP issue was the introduction of intramuscular IPV as a part of routine immunization. This introduction was announced by GOI in October 2015 with a rationale of protecting vaccine naive children by synchronously fading out the Type 2 poliovirus owing to the switch from tOPV to bOPV [7].

\section{Contribution of Indian Researchers to Polio Eradication}

India was one of the worst-hit amongst the other developing countries before the 1990s, but our scientists were proactively involved in various aspects of polio research like epidemiology and vaccine efficacy studies.
India was one of the worst-hit amongst the other developing countries before the 1990s, but our scientists were proactively involved in various aspects of polio research like epidemiology and vaccine efficacy studies. The Indian Council of Medical Research (ICMR) had established a research centre dedicated exclusively to polio related research in 1949 in Bombay (now Mumbai). It was named the Polio Research Unit. This establishment proved the farsightedness of Indian researchers. It was later renamed as the Enterovirus Research Centre (EVRC). Scientists working here actively collected the epidemiological data relating to poliomyelitis and analysed it giving valuable insights to shape up the eradication strategies. The first director of ICMR Dr C G Pandit made the first attempt to isolate poliovirus when he was studying the polio outbreak in Andaman in the late 1950s. Indian research on polio was further facilitated by the establishment of a second polio research unit called the Enterovirus Laboratory in 1964, at 
Christian Medical College, Vellore. Extensive studies carried out at both these institutes highlighted the fact that polio was hyperendemic in India. In 1966, Sabin donated his OPV strains to Pasteur Institute based in Coonoor, Tamil Nadu, and trained the staff personally to manufacture OPV. Thus, India was self-reliant in manufacturing OPV from 1968 to 1974 . However, the unit closed down as per the directives from GOI. The first breakthrough research highlighting the low efficacy of OPV was systematically studied at Vellore in 1972. These studies proved instrumental in dealing with the VAPP problem in India in the early 1990s. All this work was published way before the Expanded Program on Immunization (EPI) launched by WHO was adopted in India [3]. Indian studies were also crucial in highlighting the need to introduce IPV as a part of routine immunization. In this manner, Indian scientists successfully established early leadership in understanding the nuances of poliomyelitis especially concerning its epidemiological aspects, and thereby, made a huge scientific contribution towards advising proper intervention strategies which guided the polio eradication program. As a result of the dedication, sincerity and meticulous work done by all visionary Indian scientists, and the front-line workers along with Government of India and WHO, two decades later, India recorded its last polio case in January 2011, and was officially declared polio-free on 27 March 2014.

\section{The Work Must Continue}

Six years of being polio-free has not stopped our country from ensuring that our children stay protected from the disease. India has to stay vigilant until polio is eradicated globally, owing to the risk of re-introduction of wild polioviruses from the neighbouring countries like Pakistan and Afghanistan, which are still polio-endemic. This is being facilitated by enhancing strong surveillance programs to check on any reported paralysis case in children and the strengthening of the routine immunization programs. Emergency preparedness and response group has been constituted by the Health and Family Welfare, Government of In-
Six years of being polio-free has not stopped our country from ensuring that our children stay protected from the disease. India has to stay vigilant until polio is eradicated globally, owing to the risk of re-introduction of wild polioviruses from the neighbouring countries like Pakistan and Afghanistan, which are still polio-endemic. 
dia, to respond rapidly in case of polio re-infection. Environmental sewage sampling has also been initiated as a part of the environmental surveillance program, wherein, scientists collect sewage samples from various locations from time to time and test these samples for the presence of the poliovirus. In case of a positive result, health authorities are notified and instructed to take necessary actions which include immunization of all the children living in the area.

\section{Future of India's Polio Free Status in the Light of Covid-19} Pandemic

India's dream of staying polio-free forever seems to be facing a threat due to the recent Covid-19 pandemic. In the fight against Covid-19, governments worldwide have adopted the strategies of social distancing and countrywide lockdowns to contain SARS CoV-2. But this has created an obstacle in continuing the surveillance programs in our country, which are very crucial, given the fact that poliovirus is still endemic in our neighbouring countries. Nonetheless, our government's strategy of imposing travel restrictions, closing country, state, and district borders and physical distancing to slow Covid-19 will hopefully help suppress the spread of polio as well. In conclusion, GPEI's slogan "Every Last Child", which aims at global eradication of polio will have to be remembered for another few years by countries worldwide to achieve the polio endgame.

\section{Suggested Reading}

[1] Hamborsky J, Kroger A, Wolfe S, Center for Disease Control and Prevention, Epidemiology and Prevention of Vaccine-Preventable Diseases, eds. 13th ed. Washington D.C. Public Health Foundation, 2015.

[2] Jd Trask, A J Vignec, Paul Jr, Poliomyelitis virus in human stools, Jama., 111(1), pp.6-11, 1938, doi:10.1001/jama.1938.02790270008002.

[3] T J John, V M Vashishtha, Eradicating poliomyelitis: India's journey from hyperendemic to polio-free status, Indian J Med Res., Vol.137, No.5, pp.881894, 2013.

[4] Yash Paul, Polio eradication programme: A Failure, Economic and Political Weekly, 41(43/44), pp.4538-4540, 2006. 
[5] P P Jog, Goodbye switch and imminent polio victory, Indian Pediatr., Vol.53, No.4, pp.285-288, 2016, doi:10.1007/s13312-016-0837-9.

[6] Ministry of Health \& Family Welfare, Immunization Division, Minutes of the Meeting for tOPV to bOPV Switch. Available from http://emedinews.in/2016/jan/ Final-Minutes-of-meeting-on-tOPV-to-bOPVSwitch-in-India.pdf.

[7] V M Vashishtha, J Choudhary, et al., Introduction of Inactivated Poliovirus Vaccine in National Immunization Program and Polio Endgame Strategy, Indian Academy of Pediatrics (IAP) Advisory Committee on Vaccines and Immunization Practices (ACVIP), Indian Pediatr., 53 Suppl 1, pp.S65-S69, 2016.

[8] www.iple.in

[9] www.polioeradication.org

[10] https://www.cdc.gov/polio

[11] www.who.int

[12] Sophie Ochmann, Polio, Published online at OurWorldInData.org. 2017. Retrieved from https://ourworldindata.org/polio [Online Resource].

Address for Correspondence Anuja Madhekar

Department of Microbiology Fergusson College (Autonomous)

Pune, FC road, Shivaji Nagar Pune-411 004, India.

Email: anujaoke@gmail.com 